COMPLEXES AND FIRST-ROW

TRANSITION ELEMENTS 


\section{A Macmillan Chemistry Text}

Consulting Editor: Dr Peter Sykes, University of Cambridge

Other Titles of Related Interest

THE HEAVy TRANSITION ELEMENTS: $S . A$. Cotton and $F . A$. Hart 


\title{
COMPLEXES AND FIRST-ROW TRANSITION ELEMENTS
}

\author{
DAVID NICHOLLS
}

Senior Lecturer in the Department of Inorganic, Physical and Industrial Chemistry, University of Liverpool 
All rights reserved. No part of this publication may be reproduced or transmitted, in any form

or by any means, without permission

First published 1974 by

THE MACMILLAN PRESS LTD

London and Basingstoke

Associated companies in New York Dublin

Melbourne Johannesburg and Madras

SBN 333170881

ISBN 978-0-333-17088-5 ISBN 978-1-349-02335-6 (eBook)

DOI 10.1007/978-1-349-02335-6

The paperback edition of this book is sold subject to the condition that it shall not, by way of trade or otherwise, be lent, re-sold, hired out, or otherwise circulated without the publisher's prior consent in any form of binding or cover other than that in which it is published and without a similar condition including this condition being imposed on the subsequent purchaser. 
To Wendy 


\section{Preface}

Transition-inetal chemistry is very largely concerned with the chemistry of complexes and so it is fitting therefore that both subjects should be dealt with in the same textbook. We deal here with the general aspects of complexes first and then with the specific chemistry of the first-row transition elements. For several reasons this book deals only with the chemistry of the first-row transition elements at a level suitable for first- or second-year undergraduates in British universities. The chief of these is that the first member of each group of transition elements differs significantly in properties from the subsequent members, and thus vertical comparisons within the groups are less valuable than horizontal ones. Further, the explanations of the magnetic and spectral properties are more simply dealt with in the first-row transition elements and the aqueous chemistry can be systematised more easily. The second- and third-row transition elements are covered in a companion volume (S. A. Cotton and F. A. Hart: The Heavy Transition Elements), which also gives a more detailed treatment of organometallic compounds and the bonding therein (as well as other topics such as metal carbonyls and metal-metal bonding) at a level more appropriate to final-year honours students in British universities.

In the present text a little basic physical chemistry is assumed, in particular the concepts of atomic orbitals, elementary kinetics and thermodynamics and electrode potentials. Throughout the text emphasis is placed on the properties and reactions of metal aquo-ions and the donor-acceptor properties of transition-metal compounds. In the author's opinion a knowledge of these concepts can give the student a general understanding of the more elementary reactions of transition-metal compounds. 


\section{Contents}

Preface

1 The Development of Co-ordination Chemistry 1

1.1 Introduction to Complexes 1

1.2 The Historical Development of Co-ordination Chemistry 2

$\begin{array}{ll}\text { 1.2.1 The Werner theory } & 3\end{array}$

$\begin{array}{ll}1.2 .2 \text { Ionisable chloride } & 3\end{array}$

1.2.3 Molar conductivity 4

1.2.4 Werner's postulates 4

1.3 Nomenclature of Co-ordination Compounds 6

2 Lewis Acids and Bases 9

2.1 The Simple Lewis Approach 9

2.1.1 Types of Lewis acid 9

2.2 Types of Lewis Base (Ligands) 11

2.3 Hard and Soft Acids and Bases 14

3 The Preparation and Stability of Complex Compounds 17

$\begin{array}{ll}\text { 3.1 The Preparation of Complexes } & 17\end{array}$

3.1.1 The hexaquo-ion; aqueous substitution reactions 17

3.1.2 The hexaquo-ion; Lowry-Brönsted acidity 20

3.1.3 Preparation of complexes by direct reactions 21

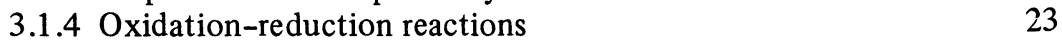

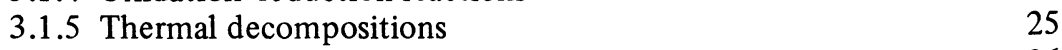

3.1.6 Metal carbonyls and organometallic compounds $\quad 26$

3.2 The Stability of Complex Ions in Solution 28

$\begin{array}{ll}3.2 .1 \text { Stability constants } & 28\end{array}$

$\begin{array}{ll}3.2 .2 \text { Stability trends } & 31\end{array}$

Further Reading $\quad 33$

4 Co-ordination Numbers and Stereochemistry 34

4.1 Valence-Shell Electron-Pair Repulsion (VSEPR) Theory 34

4.2 Co-ordination Numbers and Stereochemistries 36

4.2.1 Co-ordination numbers two and three $\quad 36$

$\begin{array}{ll}\text { 4.2.2 Co-ordination number four } & 37\end{array}$

$\begin{array}{ll}4.2 .3 \text { Co-ordination number five } & 38\end{array}$

$\begin{array}{ll}4.2 .4 \text { Co-ordination number six } & 40\end{array}$

4.2.5 Co-ordination numbers greater than six $\quad 43$

4.3 Isomerism in Co-ordination Compounds 44

4.3.1 Geometrical isomerism 44

4.3.2 Optical isomerism $\quad 45$

4.3.3 Other types of isomerism in complexes 47 
5 Theories of Bonding in Complex Compounds 48

5.1 Magnetic Properties of Complexes 48

5.2 The Valence-Bond Theory 49

5.3 The Molecular-Orbital Theory 51

5.4 The Crystal-Field Theory $\quad 56$

$\begin{array}{ll}\text { 5.4.1 Octahedral complexes } & 57\end{array}$

$\begin{array}{ll}\text { 5.4.2 Tetrahedral complexes } & 59\end{array}$

5.4.3 Crystal-field splittings in stereochemistries other than
octahedral or tetrahedral

5.5 Ligand-Field Theory in Application $\quad 62$

5.5.1 High- and low-spin complexes $\quad 62$

5.5.2 The magnitude of $\Delta$; the spectrochemical series 64

$\begin{array}{ll}\text { 5.5.3 Crystal-field stabilisation energies } & 66\end{array}$

$\begin{array}{ll}\text { 5.5.4 Stabilities of hexaquo-ions } & 67\end{array}$

$\begin{array}{ll}\text { 5.5.5 Octahedral or tetrahedral co-ordination? } & 67\end{array}$

5.5.6 Distortions from perfect symmetry; the Jahn-Teller effect $\quad 70$

6 Electronic Spectra of Transition-Metal Complexes 73

$\begin{array}{ll}6.1 \text { Introduction } & 73\end{array}$

$\begin{array}{ll}\text { 6.1.1 Ligand spectra } & 74\end{array}$

$\begin{array}{ll}\text { 6.1.2 Counter-ion spectra } & 74\end{array}$

$\begin{array}{ll}\text { 6.1.3 Charge-transfer spectra } & 74\end{array}$

$\begin{array}{ll}\text { 6.2 Ligand-Field Spectra } & 77\end{array}$

6.2.1 Terms and Russell-Saunders states $\quad 77$

$\begin{array}{ll}6.2 .2 \text { Selection rules } & 81\end{array}$

$\begin{array}{ll}\text { 6.2.3 Terms arising in ligand fields } & 83\end{array}$

$\begin{array}{ll}\text { 6.2.4 Spectra of } \mathrm{d}^{1} \text { and } \mathrm{d}^{9} \text { ions } & 85\end{array}$

6.2.5 Spectra of $\mathrm{d}^{2}$ and $\mathrm{d}^{8}$ ions $\quad 89$

$\begin{array}{ll}\text { 6.2.6 Spectra of } d^{3} \text { and } d^{7} \text { ions } & 95\end{array}$

6.2.7 Spectra of $\mathrm{d}^{5}$ ions $\quad 97$

7 Magnetic Properties of Transition-Metal Complexes 100

$\begin{array}{ll}7.1 \text { Introduction } & 100\end{array}$

7.1.1 Determination of susceptibility; the Gouy method 102

$\begin{array}{ll}7.2 \text { Orbital contribution to magnetic moments } & 103\end{array}$

7.2.1 Magnetic properties of complexes with A and E ground terms 104

7.2.2 Magnetic properties of complexes with T ground terms 106

$\begin{array}{ll}7.3 \text { High-spin-Low-spin Equilibria } & 109\end{array}$

$\begin{array}{ll}\text { 7.3.1 Thermal equilibria between spin states } & 109\end{array}$

$\begin{array}{ll}\text { 7.3.2 Structural equilibria between spin states } & 110\end{array}$

8 Mechanisms of Complex-Ion Reactions 112

$\begin{array}{lr}8.1 \text { Introduction } & 112\end{array}$

$\begin{array}{ll}8.1 .1 \text { Inert and labile complexes } & 112\end{array}$

$\begin{array}{ll}\text { 8.2 Substitution Reactions of Metal Complexes } & 113\end{array}$

8.2.1 Substitution reactions of octahedral complexes 114

8.2.2 Substitution reactions of square complexes 115 
8.3 Oxidation-Reduction Reactions

8.3.1 Outer-sphere reactions

8.3.2 Inner-sphere reactions

9 Introduction to the First-Row Transition Elements 120

9.1 Introduction

9.2 Oxidation states

9.2.1 High oxidation states

9.2.2 Low oxidation states

9.2.3 Ionic and basic character

9.3 Physical and Chemical Properties of the Elements

9.3.1 Physical properties

9.3.2 Chemical properties

10 General Preparative Methods

10.1 Introduction

10.2 Occurrence and Extraction of the Transition Metals

10.2.1 Occurrence of elements in the earth's crust

10.2.2 Extraction from oxides by thermal methods

10.2.3 Extraction from halides

10.2.4 Electrolytic methods

10.3 Preparation of Simple Transition-Metal Compounds

10.3.1 Preparation of halides

10.3.2 Preparation of nitrates

10.3.3 Preparation of other oxo-salts

10.3.4 Preparation of amides, imides, and nitrides

11 Titanium

11.1 The Element

11.2 Compounds of Titanium(IV) $\left(\mathrm{d}^{0}\right)$

11.2.1 Aqueous chemistry

11.2.2 Titanium(IV) oxide, $\mathrm{TiO}_{2} \quad 140$

11.2.3 Titanium(IV) halides

11.3 Compounds of Titanium(III) ( $\left.\mathrm{d}^{1}\right)$

11.3.1 Aqueous chemistry

11.3.2 Titanium(III) halides

11.4 Compounds of Titanium in Lower Oxidation states

11.5 Organometallic Compounds of Titanium

12.2.1 Vanadium(V) oxide and its aqueous chemistry 
12.4 Compounds of Vanadium(III) $\left(\mathrm{d}^{2}\right) \quad 154$

$\begin{array}{ll}12.4 .1 \text { Aqueous chemistry } & 154\end{array}$

12.4.2 Vanadium(III) halides $\quad 155$

$\begin{array}{ll}12.5 \text { Compounds of Vanadium(II) }\left(\mathrm{d}^{3}\right) & 156\end{array}$

$\begin{array}{ll}12.6 \text { Compounds of Vanadium(I), (0) and (-I) } & 157\end{array}$

12.6.1 Carbonyl and nitrosyl complexes 157

12.6.2 Other low oxidation state complexes $\quad 157$

$\begin{array}{ll}12.7 \text { Organometallic Compounds of Vanadium } & 158\end{array}$

13 Chromium 159

$\begin{array}{ll}\text { 13.1 The Element } & 159\end{array}$

13.2 Compounds of Chromium(VI) $\left(\mathrm{d}^{0}\right) \quad 159$

$\begin{array}{ll}\text { 13.2.1 Aqueous chemistry } & 159\end{array}$

13.2.2 Halides and oxide halides 161

13.3 Compounds of Chromium(v) $\left(\mathrm{d}^{1}\right)$ and Chromium(IV) $\left(\mathrm{d}^{2}\right) \quad 161$

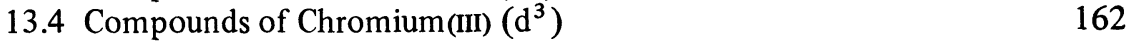

$\begin{array}{ll}\text { 13.4.1 Aqueous chemistry } & 162\end{array}$

$\begin{array}{ll}\text { 13.4.2 Chromium(III) oxide } & 163\end{array}$

13.4.3 Chromium(III) halides 163

$\begin{array}{ll}13.5 \text { Compounds of Chromium(II) }\left(\mathrm{d}^{4}\right) & 164\end{array}$

13.6 Compounds of Chromium in Low Oxidation States 165

$\begin{array}{ll}13.7 \text { Organometallic Compounds of Chromium } & 166\end{array}$

14 Manganese 167

$\begin{array}{ll}\text { 14.1 The Element } & 167\end{array}$

14.2 Compounds of Manganese(VII) $\left(\mathrm{d}^{0}\right)$, Manganese(VI) $\left(\mathrm{d}^{1}\right)$, and Manganese $(\mathrm{V})\left(\mathrm{d}^{2}\right) \quad 167$

$\begin{array}{ll}14.3 \text { Compounds of Manganese(IV) }\left(\mathrm{d}^{3}\right) & 168\end{array}$

$\begin{array}{ll}14.4 \text { Compounds of Manganese(III) }\left(\mathrm{d}^{4}\right) & 169\end{array}$

$\begin{array}{ll}14.5 \text { Compounds of Manganese(II) }\left(\mathrm{d}^{5}\right) & 170\end{array}$

$\begin{array}{ll}\text { 14.6 Compounds of Manganese in Low Oxidation States } & 171\end{array}$

$\begin{array}{ll}14.7 \text { Organometallic Compounds of Manganese } & 171\end{array}$

15 Iron 172

$\begin{array}{ll}\text { 15.1 The Element } & 172\end{array}$

15.2 Compounds of Iron in High Oxidation States 173

$\begin{array}{ll}15.3 \text { Compounds of Iron(III) }\left(\mathrm{d}^{5}\right) & 174\end{array}$

$\begin{array}{ll}\text { 15.3.1 Aqueous chemistry } & 174\end{array}$

$\begin{array}{ll}\text { 15.3.2 Simple compounds } & 176\end{array}$

$\begin{array}{ll}15.4 \text { Compounds of Iron(II) }\left(\mathrm{d}^{6}\right) & 177\end{array}$

$\begin{array}{ll}\text { 15.4.1 Aqueous chemistry } & 178\end{array}$

$\begin{array}{ll}\text { 15.4.2 Some simple compounds } & 178\end{array}$

$\begin{array}{ll}15.4 .3 \text { Complexes } & 179\end{array}$

15.5 Compounds of Iron in Low Oxidation States 181

$\begin{array}{ll}\text { 15.5.1 Iron Carbonyls } & 181\end{array}$

15.5.2 Nitrogen monoxide complexes of iron 183

$\begin{array}{ll}15.6 \text { Organometallic Compounds of Iron } & 183\end{array}$ 
16 Cobalt 185

$\begin{array}{ll}\text { 16.1 The Element } & 185\end{array}$

$\begin{array}{ll}\text { 16.2 Compounds of Cobalt(III) }\left(\mathrm{d}^{6}\right) & 185\end{array}$

16.2.1 Simple compounds 186

16.2.2 Complex compounds 186

16.3 Compounds of Cobalt(II) $\left(\mathrm{d}^{7}\right) \quad 188$

$\begin{array}{ll}\text { 16.3.1 Aqueous chemistry } & 188\end{array}$

$\begin{array}{ll}\text { 16.3.2 Simple salts } & 188\end{array}$

$\begin{array}{ll}\text { 16.3.3 Complexes } & 189\end{array}$

16.4 Compounds of Cobalt in Low Oxidation States 191

$\begin{array}{ll}\text { 16.4.1 Cobalt carbonyls } & 191\end{array}$

16.4.2 Cobalt nitrosyls 192

16.4.3 Other low oxidation state compounds 192

$\begin{array}{ll}16.5 \text { Organometallic chemistry of cobalt } & 192\end{array}$

17 Nickel 194

$\begin{array}{ll}\text { 17.1 The Element } & 194\end{array}$

17.2 Compounds of Nickel in High Oxidation States 194

$\begin{array}{ll}17.3 \text { Compounds of Nickel(II) }\left(\mathrm{d}^{8}\right) & 195\end{array}$

$\begin{array}{ll}\text { 17.3.1 Aqueous chemistry } & 195\end{array}$

$\begin{array}{ll}\text { 17.3.2 Simple compounds } & 195\end{array}$

$\begin{array}{ll}\text { 17.3.3 Complexes } & 196\end{array}$

17.4 Compounds of Nickel in Low Oxidation States 198

$\begin{array}{ll}\text { 17.4.1 Nickel carbonyl } & 198\end{array}$

$\begin{array}{ll}\text { 17.4.2 Nickel nitrosyls } & 199\end{array}$

17.4.3 Other low oxidation state compounds 199

$\begin{array}{ll}17.5 \text { Organometallic compounds of nickel } & 200\end{array}$

18 Copper 201

$\begin{array}{ll}\text { 18.1 The Element } & 201\end{array}$

$\begin{array}{ll}18.2 \text { Compounds of Copper(III) }\left(\mathrm{d}^{8}\right) & 201\end{array}$

$\begin{array}{ll}18.3 \text { Compounds of Copper(II) }\left(\mathrm{d}^{9}\right) & 201\end{array}$

$\begin{array}{ll}\text { 18.3.1 Aqueous chemistry } & 202\end{array}$

18.3.2 Simple compounds 203

18.4 Compounds of Copper(I) $\left(\mathrm{d}^{10}\right) \quad 204$

$\begin{array}{ll}\text { 18.4.1 Aqueous chemistry } & 204\end{array}$

$\begin{array}{ll}\text { 18.4.2 Simple compounds } & 205\end{array}$

18.4.3 Carbonyls and organometallic compounds 206

$\begin{array}{lr}\text { Bibliography } & 207\end{array}$

$\begin{array}{ll}\text { Index } & 211\end{array}$ 